Adv. Studies Theor. Phys., Vol. 8, 2014, no. 6, 285 - 292

HIKARI Ltd, www.m-hikari.com

http://dx.doi.org/10.12988/astp.2014.4218

\title{
Symmetric Identities for the Generalized Higher-order $q$-Bernoulli Polynomials
}

\author{
Dae San Kim \\ Department of Mathematics, Sogang University \\ Seoul 121-742, Republic of Korea \\ Taekyun Kim \\ Department of Mathematics, Kwangwoon University \\ Seoul 139-701, Republic of Korea \\ Sang-Hun Lee \\ Division of General Education, Kwangwoon University \\ Seoul 139-701, Republic of Korea
}

Copyright (c) 2014 Dae San Kim, Taekyun Kim and Sang-Hun Lee. This is an open access article distributed under the Creative Commons Attribution License, which permits unrestricted use, distribution, and reproduction in any medium, provided the original work is properly cited.

\begin{abstract}
In this paper, we give identities of symmetry for the generalized higher-order $q$ Bernoulli polynomials attached to $\chi$ which are derived from the symmetric properties of multivariate $p$-adic invariant integrals on $\mathbb{Z}_{p}$.
\end{abstract}

\section{INTRODUCTION}

As is well known, the higher order Bernoulli polynomials are defined by the generating function to be

$$
\left(\frac{t}{e^{t}-1}\right)^{r} e^{x t}=\sum_{n=0}^{\infty} B_{n}^{(r)}(x) \frac{t^{n}}{n !}, \quad(n \in \mathbb{N}), \quad(\text { see }[1,4,11])
$$


When $x=0, B_{n}^{(r)}=B_{n}^{(r)}(0)$ is called the $n$-th Bernoulli number of order $r$. Let $\chi$ be a primitive Dirichlet character with conductor $d \in \mathbb{N}$. Then the generalized Bernoulli polynomials attached to $\chi$ are defined by the generating function to be

$$
\left(\frac{t}{e^{d t}-1} \sum_{a=0}^{d-1} \chi(a) e^{a t}\right) e^{x t}=\sum_{n=0}^{\infty} B_{n, \chi}(x) \frac{t^{n}}{n !}, \quad(\text { see }[5,6]) .
$$

For $r \in \mathbb{N}$, in view of (1), we may also consider the generalized higher-order Bernoulli polynomials attached to $\chi$ as follows:

$$
\left(\frac{t \sum_{a=0}^{d-1} \chi(a) e^{a t}}{e^{d t}-1}\right)^{r} e^{x t}=\sum_{n=0}^{\infty} B_{n, \chi}^{(r)}(x) \frac{t^{n}}{n !} .
$$

When $x=0, B_{n, \chi}^{(r)}=B_{n, \chi}^{(r)}(0)$ is called the $n$-th generalized Bernoulli numbers attached to $\chi$ with order $r$. Let $p$ be a fixed prime number. Throughout this paper $\mathbb{Z}_{p}, \mathbb{Q}_{p}$, and $\mathbb{C}_{p}$ will, respectively, denote the ring of $p$-adic rational integers, the field of $p$-adic rational numbers and the completion of algebraic closure of $\mathbb{Q}_{p}$. Let $\nu_{p}$ be the normalized exponential valuation of $\mathbb{C}_{p}$ with $|p|_{p}=$ $p^{-\nu_{p}(p)}=\frac{1}{p}$. When one talks about $q$-extensions, $q$ is variously considered as an indeterminate, a complex number $q \in \mathbb{C}$, or a $p$-adic number $q \in \mathbb{C}_{p}$. If $q \in \mathbb{C}$, one usually assumes $|q|<1$;if $q \in \mathbb{C}_{p}$, one usually assumes $|1-q|_{p}<p^{-\frac{1}{p-1}}$ so that $q^{x}=\exp (x \log q)$ for $|x|_{p} \leq 1$. The $q$-number of $x$ is defined by $[x]_{q}=\frac{1-q^{x}}{1-q}$. Note that $\lim _{q \rightarrow 1}[x]_{q}=x$. Let $U D\left(\mathbb{Z}_{p}\right)$ be the space of uniformly differentiable functions on $\mathbb{Z}_{p}$. For $f \in U D\left(\mathbb{Z}_{p}\right)$, the $p$-adic invariant integral on $\mathbb{Z}_{p}$ is defined by

$$
I_{0}(f)=\int_{\mathbb{Z}_{p}} f(x) d \mu_{0}(x)=\lim _{N \rightarrow \infty} \frac{1}{p^{N}} \sum_{x=0}^{p^{N}-1} f(x), \quad \text { (see [1-11]). }
$$

From (4), we note that

$$
I_{0}\left(f_{1}\right)=I_{0}(f)+f^{\prime}(0) \text {, where } f_{1}(x)=f(x+1) .
$$

Thus, by (5), we get

$$
I_{0}\left(f_{n}\right)=I_{0}(f)+\sum_{l=0}^{n-1} f^{\prime}(l)
$$

where $n \in \mathbb{N}$ and $f_{n}(x)=f(x+n)$.

Let $d$ be a fixed positive integer and let $p$ be a fixed prime number. For $N \in \mathbb{N}$, we set 


$$
\begin{gathered}
X=\lim _{\overleftarrow{N}}\left(\mathbb{Z} / d p^{N} \mathbb{Z}\right), \quad X^{*}=\bigcup_{\substack{0<a<d p \\
(a, p)=1}} a+d p \mathbb{Z}_{p}, \\
a+d p^{N} \mathbb{Z}_{p}=\left\{x \in X \mid x \equiv a \quad\left(\bmod d p^{N}\right)\right\},
\end{gathered}
$$

where $a \in \mathbb{Z}$ lies in $0 \leq a<d p^{N}$.

It is not difficult to show that

$$
\int_{X} f(x) d \mu_{0}(x)=\int_{\mathbb{Z}_{p}} f(x) d \mu_{0}(x),
$$

where $f(x) \in U D\left(\mathbb{Z}_{p}\right)$, (see $\left.[5,6,7]\right)$.

Let us take $f(x)=e^{x t} \chi(x)$. Then, by (6), we get

$$
\int_{X} \chi(x) e^{x t} d \mu_{0}(x)=\frac{t}{e^{d t}-1} \sum_{a=0}^{d-1} \chi(a) e^{a t}=\sum_{n=0}^{\infty} B_{x, \chi} \frac{t^{n}}{n !} .
$$

By (8), we see that

$$
\begin{aligned}
\int_{X} \chi(y) e^{(x+y) t} d \mu_{0}(y) & =\left(\frac{t}{e^{d t}-1} \sum_{a=0}^{d-1} \chi(a) e^{a t}\right) e^{x t} \\
& =\sum_{n=0}^{\infty} B_{n, \chi}(x) \frac{t^{n}}{n !} .
\end{aligned}
$$

From (9), we can derive the following equation (10):

$$
\begin{aligned}
& \int_{X} \ldots \int_{X}\left(\Pi_{l=1}^{r} \chi\left(y_{l}\right)\right) e^{\left(x+y_{1}+\cdots+y_{r}\right) t} d \mu_{0}\left(y_{1}\right) \ldots d \mu_{0}\left(y_{r}\right) \\
& =\left(\frac{t \sum_{a=0}^{d-1} \chi(a) e^{a t}}{e^{d t}-1}\right)^{r} e^{x t}=\sum_{n=0}^{\infty} B_{n, \chi}^{(r)}(x) \frac{t^{n}}{n !} .
\end{aligned}
$$

In the next section, we consider $q$-extensions of (10). The purpose of this paper is to give identities of symmetry for the generalized $q$-Bernoulli polynomials of order $r$ which are derived from the symmetric properties of multivariate $p$ adic invariant integrals on $\mathbb{Z}_{p}$.

\section{Symmetric identities of The GenERALIZEd HigheR-ORDER $q$-BERNOULLI POLYNOMIALS}

Now, we consider the generalized higher-order $q$-Bernoulli polynomials attached to $\chi$ with the viewpoint of (10) as follows: 


$$
\begin{aligned}
& \int_{X} \ldots \int_{X}\left(\Pi_{l=1}^{r} \chi\left(y_{l}\right)\right) e^{\left[x+y_{1}+\cdots+y_{r}\right]_{q} t} d \mu_{0}\left(y_{1}\right) \ldots d \mu_{0}\left(y_{r}\right) \\
& =\sum_{n=0}^{\infty} B_{n, \chi, q}^{(r)}(x) \frac{t^{n}}{n !} .
\end{aligned}
$$

Thus, by (11), we get

$$
\begin{gathered}
B_{n, \chi, q}^{(r)}(x)=\int_{X} \ldots \int_{X}\left(\Pi_{l=1}^{r} \chi\left(y_{l}\right)\right)\left[x+y_{1}+\cdots+y_{r}\right]_{q}^{n} d \mu_{0}\left(y_{1}\right) \ldots d \mu_{0}\left(y_{r}\right) \\
=\frac{[d]_{q}^{n}}{d^{r}} \sum_{a_{1}, \cdots, a_{r}=0}^{d-1}\left(\Pi_{l=1}^{r} \chi\left(a_{l}\right)\right) \\
\times \int_{\mathbb{Z}_{p}} \cdots \int_{\mathbb{Z}_{p}}\left[\frac{x+a_{1}+\cdots+a_{r}}{d}+y_{1}+\cdots+y_{r}\right]_{q^{d}}^{n} d \mu_{0}\left(y_{1}\right) \ldots d \mu_{0}\left(y_{r}\right) \\
=\frac{[d]_{q}^{n}}{d^{r}} \sum_{a_{1}, \cdots, a_{r}=0}^{d-1}\left(\prod_{l=1}^{r} \chi\left(a_{l}\right)\right) B_{n, q^{d}}^{(r)}\left(\frac{x+a_{1}+\cdots+a_{r}}{d}\right)
\end{gathered}
$$

where $B_{n, q}^{(r)}(x)$ are higher-order $q$-Bernoulli polynomials which are defined by

$$
B_{n, q}^{(r)}(x)=\int_{\mathbb{Z}_{p}} \ldots \int_{\mathbb{Z}_{p}}\left[x+y_{1}+\cdots+y_{r}\right]_{q}^{n} d \mu_{0}\left(y_{1}\right) \ldots d \mu_{0}\left(y_{r}\right) .
$$

From (12), we note that

$$
B_{n, \chi, q}^{(r)}(x)=\sum_{l=0}^{n}\left(\begin{array}{l}
n \\
l
\end{array}\right)[x]_{q}^{n-l} q^{l x} B_{l, \chi, q}^{(r)}
$$

where $B_{l, \chi, q}^{(r)}=B_{l, \chi, q}^{(r)}(0)$ is the $l$-th generalized higher-order $q$-Bernoulli number attached to $\chi$.

Let $w_{1}, w_{2}$ be natural numbers. Then, by (11), we get

$$
\begin{gathered}
\frac{1}{w_{1}^{r}} \int_{X} \ldots \int_{X}\left(\Pi_{l=1}^{r} \chi\left(y_{l}\right)\right) e^{\left[w_{1} w_{2} x+w_{2} \sum_{l=1}^{r} j_{l}+w_{1} \sum_{l=1}^{r} y_{l}\right]_{q} t} d \mu_{0}\left(y_{1}\right) \ldots d \mu_{0}\left(y_{r}\right) \\
=\lim _{N \rightarrow \infty}\left(\frac{1}{d w_{1} w_{2} p^{N}}\right)^{r} \sum_{i_{1}, \cdots, i_{r}=0}^{d w_{2}-1}\left(\prod_{l=1}^{r} \chi\left(i_{l}\right)\right) \\
\times \sum_{y_{1}, \cdots, y_{r}=0}^{p^{N}-1} e^{\left[w_{1} w_{2} x+w_{2} \sum_{l=1}^{r} j_{l}+w_{1} \sum_{l=1}^{r}\left(i_{l}+d w_{2} y_{l}\right)\right]_{q} t}
\end{gathered}
$$

Thus, by (14), we get 


$$
\begin{aligned}
& \frac{1}{w_{1}^{r}} \sum_{j_{1}, \cdots, j_{r}=0}^{d w_{1}-1}\left(\Pi_{l=1}^{r} \chi\left(j_{l}\right)\right) \int_{X} \cdots \int_{X}\left(\Pi_{l=1}^{r} \chi\left(y_{l}\right)\right) \\
& \times e^{\left[w_{1} w_{2} x+w_{2} \sum_{l=1}^{r} j_{l}+w_{1} \sum_{l=1}^{r} y_{l}\right]_{q} t} d \mu_{0}\left(y_{1}\right) \ldots d \mu_{0}\left(y_{r}\right) \\
& =\lim _{N \rightarrow \infty}\left(\frac{1}{d w_{1} w_{2} p^{N}}\right)^{r} \sum_{y_{1}, \cdots, y_{r}=0}^{p^{N}-1} \sum_{j_{1}, \cdots, j_{r}=0}^{d w_{1}-1} \sum_{i_{1}, \cdots, i_{r}=0}^{d w_{2}-1}\left(\Pi_{l=1}^{r} \chi\left(i_{l}\right) \chi\left(j_{l}\right)\right) \\
& \times e^{\left[w_{1} w_{2} x+w_{2} \sum_{l=1}^{r} j_{l}+w_{1} \sum_{l=1}^{r}\left(i_{l}+d w_{2} y_{l}\right)\right]_{q} t}
\end{aligned}
$$

By the same method as (15), we get

$$
\begin{aligned}
& \frac{1}{w_{2}^{r}} \sum_{j_{1}, \cdots, j_{r}=0}^{d w_{2}-1}\left(\Pi_{l=1}^{r} \chi\left(j_{l}\right)\right) \int_{X} \cdots \int_{X}\left(\Pi_{l=1}^{r} \chi\left(y_{l}\right)\right) \\
& \times e^{\left[w_{1} w_{2} x+w_{1} \sum_{l=1}^{r} j_{l}+w_{2} \sum_{l=1}^{r} y_{l}\right]_{q} t} d \mu_{0}\left(y_{1}\right) \ldots d \mu_{0}\left(y_{r}\right) \\
& =\lim _{N \rightarrow \infty}\left(\frac{1}{d w_{1} w_{2} p^{N}}\right)^{r} \sum_{y_{1}, \cdots, y_{r}=0}^{p^{N}-1} \sum_{j_{1}, \cdots, j_{r}=0}^{d w_{2}-1} \sum_{i_{1}, \cdots, i_{r}=0}^{d w_{1}-1}\left(\Pi_{l=1}^{r} \chi\left(i_{l}\right) \chi\left(j_{l}\right)\right) \\
& \times e^{\left[w_{1} w_{2} x+w_{1} \sum_{l=1}^{r} j_{l}+w_{2} \sum_{l=1}^{r}\left(i_{l}+d w_{2} y_{l}\right)\right]_{q} t}
\end{aligned}
$$

Therefore, by (15) and (16), we obtain the following theorem.

Theorem 2.1. For $w_{1}, w_{2}, d \in \mathbb{N}$, we have

$$
\begin{aligned}
& \frac{1}{w_{1}^{r}} \sum_{j_{1}, \cdots, j_{r}=0}^{d w_{1}-1}\left(\Pi_{l=1}^{r} \chi\left(j_{l}\right)\right) \int_{X} \ldots \int_{X}\left(\Pi_{l=1}^{r} \chi\left(y_{l}\right)\right) \\
& \quad \times e^{\left[w_{1} w_{2} x+w_{2} \sum_{l=1}^{r} j_{l}+w_{1} \sum_{l=1}^{r} y_{l}\right]_{q} t} d \mu_{0}\left(y_{1}\right) \ldots d \mu_{0}\left(y_{r}\right) \\
& =\frac{1}{w_{2}^{r}} \sum_{j_{1}, \cdots, j_{r}=0}^{d w_{2}-1}\left(\Pi_{l=1}^{r} \chi\left(j_{l}\right)\right) \int_{X} \ldots \int_{X}\left(\prod_{l=1}^{r} \chi\left(y_{l}\right)\right) \\
& \quad \times e^{\left[w_{1} w_{2} x+w_{1} \sum_{l=1}^{r} j_{l}+w_{2} \sum_{l=1}^{r} y_{l}\right]_{q} t} d \mu_{0}\left(y_{1}\right) \ldots d \mu_{0}\left(y_{r}\right)
\end{aligned}
$$

Note that

$$
\left[w_{1} w_{2} x+w_{2} \sum_{l=1}^{r} j_{l}+w_{1} \sum_{l=1}^{r} y_{l}\right]_{q}=\left[w_{1}\right]_{q}\left[w_{2} x+\frac{w_{2}}{w_{1}} \sum_{l=1}^{r} j_{l}+\sum_{l=1}^{r} y_{l}\right]_{q^{w_{1}}},
$$

and

$$
\left[w_{1} w_{2} x+w_{1} \sum_{l=1}^{r} j_{l}+w_{2} \sum_{l=1}^{r} y_{l}\right]_{q}=\left[w_{2}\right]_{q}\left[w_{1} x+\frac{w_{1}}{w_{2}} \sum_{l=1}^{r} j_{l}+\sum_{l=1}^{r} y_{l}\right]_{q^{w_{2}}} .
$$

Therefore, by Theorem 2.1, (17) and (18), we obtain the following Corollary. 
Corollary 2.2. For $n \geq 0$, we have

$$
\begin{aligned}
& \frac{\left[w_{1}\right]_{q}^{n}}{w_{1}^{r}} \sum_{j_{1}, \cdots, j_{r}=0}^{d w_{1}-1}\left(\Pi_{l=1}^{r} \chi\left(j_{l}\right)\right) \int_{X} \ldots \int_{X}\left(\Pi_{l=1}^{r} \chi\left(y_{l}\right)\right) \\
& \times\left[w_{2} x+\frac{w_{2}}{w_{1}} \sum_{l=1}^{r} j_{l}+\sum_{l=1}^{r} y_{l}\right]_{q^{w_{1}}}^{n} d \mu_{0}\left(y_{1}\right) \ldots d \mu_{0}\left(y_{r}\right) \\
& =\frac{\left[w_{2}\right]_{q}^{n}}{w_{2}^{r}} \sum_{j_{1}, \cdots, j_{r}=0}^{d w_{2}-1}\left(\Pi_{l=1}^{r} \chi\left(j_{l}\right)\right) \int_{X} \ldots \int_{X}\left(\Pi_{l=1}^{r} \chi\left(y_{l}\right)\right) \\
& \times\left[w_{1} x+\frac{w_{1}}{w_{2}} \sum_{l=1}^{r} j_{l}+\sum_{l=1}^{r} y_{l}\right]_{q^{w_{2}}}^{n} d \mu_{0}\left(y_{1}\right) \ldots d \mu_{0}\left(y_{r}\right)
\end{aligned}
$$

Therefore, by (12) and Corollary 2.2, we obtain the following theorem.

Theorem 2.3. For $n \geq 0, w_{1}, w_{2} \in \mathbb{N}$, we have

$$
\begin{aligned}
& \frac{\left[w_{1}\right]_{q}^{n}}{w_{1}^{r}} \sum_{j_{1}, \cdots, j_{r}=0}^{d w_{1}-1}\left(\Pi_{l=1}^{r} \chi\left(j_{l}\right)\right) B_{n, \chi, q^{w_{1}}}^{(r)}\left(w_{2} x+\frac{w_{2}}{w_{1}}\left(j_{1}+\cdots+j_{r}\right)\right) \\
= & \frac{\left[w_{2}\right]_{q}^{n}}{w_{2}^{r}} \sum_{j_{1}, \cdots, j_{r}=0}^{d w_{2}-1}\left(\Pi_{l=1}^{r} \chi\left(j_{l}\right)\right) B_{n, \chi, q^{w_{2}}}^{(r)}\left(w_{1} x+\frac{w_{1}}{w_{2}}\left(j_{1}+\cdots+j_{r}\right)\right) .
\end{aligned}
$$

From (12), we can derive the following equation (19):

$$
\begin{aligned}
& \int_{X} \ldots \int_{X}\left(\Pi_{l=1}^{r} \chi\left(y_{l}\right)\right)\left[w_{2} x+\frac{w_{2}}{w_{1}} \sum_{l=1}^{r} j_{l}+\sum_{l=1}^{r} y_{l}\right]_{q^{w_{1}}}^{n} d \mu_{0}\left(y_{1}\right) \ldots d \mu_{0}\left(y_{r}\right) \\
& =\sum_{i=0}^{n}\left(\begin{array}{c}
n \\
i
\end{array}\right)\left(\frac{\left[w_{2}\right]_{q}}{\left[w_{1}\right]_{q}}\right)^{i}\left[j_{1}+\cdots+j_{r}\right]_{q^{w_{2}}}^{i} q^{w_{2}(n-i) \sum_{l=1}^{r} j_{l}} \\
& \quad \times \int_{\mathbb{Z}_{p}} \ldots \int_{\mathbb{Z}_{p}}\left[w_{2} x+\sum_{l=1}^{r} y_{l}\right]_{q^{w_{1}}}^{n-i}\left(\prod_{l=1}^{r} \chi\left(y_{l}\right)\right) d \mu_{0}\left(y_{1}\right) \ldots d \mu_{0}\left(y_{r}\right) \\
& =\sum_{i=0}^{n}\left(\begin{array}{c}
n \\
i
\end{array}\right)\left(\frac{\left[w_{2}\right]_{q}}{\left[w_{1}\right]_{q}}\right)^{i}\left[j_{1}+\cdots+j_{r}\right]_{q^{w_{2}}}^{i} q^{w_{2}(n-i) \sum_{l=1}^{r} j_{l}} B_{n-i, \chi, q^{w_{1}}}^{(r)}\left(w_{2} x\right) .
\end{aligned}
$$

Thus, by (19), we get 


$$
\begin{aligned}
& \frac{\left[w_{1}\right]_{q}^{n}}{w_{1}^{r}} \sum_{j_{1}, \cdots, j_{r}=0}^{d w_{1}-1}\left(\Pi_{l=1}^{r} \chi\left(j_{l}\right)\right) \int_{X} \cdots \int_{X}\left(\Pi_{l=1}^{r} \chi\left(y_{l}\right)\right) \\
& \times\left[w_{2} x+\frac{w_{2}}{w_{1}} \sum_{l=1}^{r} j_{l}+\sum_{l=1}^{r} y_{l}\right]_{q^{w_{1}}}^{n} d \mu_{0}\left(y_{1}\right) \ldots d \mu_{0}\left(y_{r}\right) \\
& =\sum_{i=0}^{n}\left(\begin{array}{c}
n \\
i
\end{array}\right) \frac{\left[w_{1}\right]_{q}^{n-i}}{w_{1}^{r}}\left[w_{2}\right]_{q}^{i}\left(\sum_{j_{1}, \cdots, j_{r}=0}^{d w_{1}-1}\left[j_{1}+\cdots+j_{r}\right]_{q^{w_{2}}}^{i}\left(\Pi_{l=1}^{r} \chi\left(j_{l}\right)\right) q^{w_{2}(n-i) \sum_{l=1}^{r} j_{l}}\right) \\
& \times B_{n-i, \chi, q^{w_{1}}}^{(r)}\left(w_{2} x\right) \\
& =\sum_{i=0}^{n}\left(\begin{array}{c}
n \\
i
\end{array}\right) \frac{\left[w_{1}\right]_{q}^{i}}{w_{1}^{r}}\left[w_{2}\right]_{q}^{n-i}\left(\sum_{j_{1}, \cdots, j_{r}=0}^{d w_{1}-1}\left[j_{1}+\cdots+j_{r}\right]_{q^{w_{2}}}^{n-i} q^{w_{2} i \sum_{l=1}^{r} j_{l}}\left(\Pi_{l=1}^{r} \chi\left(j_{l}\right)\right)\right) \\
& \times B_{i, \chi, q^{w_{1}}}^{(r)}\left(w_{2} x\right) \\
& =\sum_{i=0}^{n}\left(\begin{array}{c}
n \\
i
\end{array}\right) \frac{\left[w_{1}\right]_{q}^{i}}{w_{1}^{r}}\left[w_{2}\right]_{q}^{n-i} T_{n, i}^{(r)}\left(d w_{1}, q^{w_{2}} \mid \chi\right) B_{i, \chi, q^{w_{1}}}^{(r)}\left(w_{2} x\right)
\end{aligned}
$$

where

$$
T_{n, i}^{(r)}(w, q \mid \chi)=\sum_{j_{1}, \cdots, j_{r}=0}^{w-1}\left[j_{1}+\cdots+j_{r}\right]_{q}^{n-i} q^{i \sum_{l=1}^{r} j_{l}}\left(\Pi_{l=1}^{r} \chi\left(j_{l}\right)\right) .
$$

By the same method as (20), we get

$$
\begin{aligned}
& \frac{\left[w_{2}\right]_{q}^{n}}{w_{2}^{r}} \sum_{j_{1}, \cdots, j_{r}=0}^{d w_{2}-1}\left(\Pi_{l=1}^{r} \chi\left(j_{l}\right)\right) \int_{X} \ldots \int_{X}\left(\Pi_{l=1}^{r} \chi\left(y_{l}\right)\right) \\
& \quad \times\left[w_{1} x+\frac{w_{1}}{w_{2}} \sum_{l=1}^{r} j_{l}+\sum_{l=1}^{r} y_{l}\right]_{q^{w_{2}}}^{n} d \mu_{0}\left(y_{1}\right) \ldots d \mu_{0}\left(y_{r}\right) \\
& =\sum_{i=0}^{n}\left(\begin{array}{c}
n \\
i
\end{array}\right) \frac{\left[w_{2}\right]_{q}^{i}}{w_{2}^{r}}\left[w_{1}\right]_{q}^{n-i} T_{n, i}^{(r)}\left(d w_{2}, q^{w_{1}} \mid \chi\right) B_{i, \chi, q^{w_{2}}}^{(r)}\left(w_{1} x\right) .
\end{aligned}
$$

Therefore, by (20) and (22), we obtain the following theorem.

Theorem 2.4. For $n \geq 0, w_{1}, w_{2} \in \mathbb{N}$, we have

$$
\begin{aligned}
& \sum_{i=0}^{n}\left(\begin{array}{c}
n \\
i
\end{array}\right) \frac{\left[w_{1}\right]_{q}^{i}}{w_{1}^{r}}\left[w_{2}\right]_{q}^{n-i} T_{n, i}^{(r)}\left(d w_{1}, q^{w_{2}} \mid \chi\right) B_{i, \chi, q^{w_{1}}}^{(r)}\left(w_{2} x\right) \\
= & \sum_{i=0}^{n}\left(\begin{array}{c}
n \\
i
\end{array}\right) \frac{\left[w_{2}\right]_{q}^{i}}{w_{2}^{r}}\left[w_{1}\right]_{q}^{n-i} T_{n, i}^{(r)}\left(d w_{2}, q^{w_{1}} \mid \chi\right) B_{i, \chi, q^{w_{2}}}^{(r)}\left(w_{1} x\right),
\end{aligned}
$$


where

$$
T_{n, i}^{(r)}(w, q \mid \chi)=\sum_{j_{1}, \cdots, j_{r}=0}^{w-1}\left[j_{1}+\cdots+j_{r}\right]_{q}^{n-i} q^{i \sum_{l=1}^{r} j_{l}}\left(\Pi_{l=1}^{r} \chi\left(j_{l}\right)\right)
$$

\section{REFERENCES}

[1] L. Carlitz, q-Bernoulli numbers and polynomials, Duke Math. J. 15(1948), 987-1000.

[2] M. Cenkci, V. Kurt, Congruences for generalized q-Bernoulli polynomials, J. Inequal. Appl. 2008, Art.ID 270713, 19 pp.

[3] D. V. Dolgy, T. Kim, B. Lee, S.-H. Lee, Some new identities on the twisted Bernoulli and Euler polynomials, J. Comput. Anal. Appl. 15(2013), no. 3, 441-451.

[4] D. S. Kim, N. Lee, J. Na, K. H. Park, Abundant symmetry for higher-order Bernoulli polynomials (I), Adv. Stud. Contemp. Math. 23 (2013), no. 3, 461-482.

[5] T. Kim, S.-H. Rim, Generalized Carlitz's q-Bernoulli numbers in the p-adic number field, Adv. Stud. Contemp. Math. 2 (2000), 9-19.

[6] T. Kim, On explicit formulas of p-adic q-L-functions, Khyshu J. Math. 48(1994), no. $1,73-86$.

[7] T. Kim, q-Volkenborn integration, Russ. J. Math. Phys. 9(2002), no. 3, 288-299.

[8] T. Kim, On the symmetries of the q-Bernoulli polynomials, Abstr. Appl. Anal. 2008, Art.ID 914367, 7 pp.

[9] H. Ozden, I. N. Cangul, Y. Simsek, Remarks on q-Bernoulli numbers associated with Daehee numbers, Adv. Stud. Contemp. Math. 18(2009), no.1, 41-48.

[10] H.-K. Pak, S.-H. Rim, q-Bernoulli numbers and polynomials via an invariant p-adic $q$-integral on $\mathbb{Z}_{p}$, Notes Number Thoery Discrete Math. 7(2001), no.4, 105-110.

[11] S. H. Rim, A. Bayad, E.-J. Moon, J.-H. Jin, S.-J. Lee, A new construction on the q-Bernoulli polynomials, Adv. Difference Equ. 2011, 2011:34, 6 pp.

[12] Y. Simsek, Generating functions of the twisted Bernoulli numbers and polynomials associated with their interpolation functions, Adv. Stud. Contemp. Math. 16(2008), no.2, 251-278.

\section{Received: February 1, 2014}

